\title{
Arbor
}

\section{Sobre la naturaleza histórica de la Matemática y su enseñanza}

\author{
Francisco A. González Redondo
}

Arbor CLXXIII, 681 (Septiembre 2002), 203-223 pp.

En un sistema educativo español siempre en crisis, con reformas sucesivas encadenándose a todos los niveles, la enseñanza de la Matemática -quizá de manera más llamativa que la de otras materias- mantiene constante su relevancia especial: es intrínsecamente problemática. Finalizado el 2000, pomposamente denominado «Año Mundial de las Matemáticas», la situación no sólo no se ha aclarado sino que el problema alcanza incluso al hasta ahora intocable nivel universitario. Admitidas sin discusión en el mundo de la pedagogía matemática las estrechas relaciones que existen -y deben existir-entre los procesos de aprehensión de los conceptos y métodos de la disciplina, y los asociados a la evolución que ha tenido nuestra Ciencia en la Historia, la génesis de los conocimientos y la transmisión de los mismos, en este trabajo se presenta un modelo historiográfico que podría contribuir a fijar algunos puntos del siempre vivo debate.

A modo de prólogo de este artículo me ha parecido oportuno destacar un ejemplo tomado de esa fuente inagotable de recursos didácticos que suponen los estudios históricos sobre las diferentes ciencias.

En la tablilla BM 85194, una de las conservadas en el British Museum de Londres, escrita con el simbolismo cuneiforme propio de la época babilónica de la que procede $-c$. 1800 a.C.-, y con el uso del sistema de 
numeración posicional sexagesimal desarrollado por los sabios del momento, se recogen, en tres apretadas columnas, un conjunto de 17 problemas de contenido aritmético y/o geométrico que ilustra el quehacer en la instrucción matemática usual en las escuelas de escribas de Mesopotamia (Neugebauer 1969, Plate 3), donde este tipo de materiales jugaban el papel de «libros de texto».

De entre todos ellos hay uno especialmente significativo que puede seleccionarse como ejemplo introductorio de muchas de las cuestiones que se van tratar más adelante en este trabajo. En lenguaje matemático más actual $^{1}$, el problema es el siguiente: Una ciudad, rodeada por un muro circular, se ha expansionado en todas las direcciones de tal modo que resulta necesario construir un nuevo cierre, también circular, que rodee las nuevas edificaciones. Los datos que se proporcionan son la distancia entre los dos muros (5, sin precisar unidades de longitud) y el área entre ambos (análogamente en el sistema sexagesimal, sin referencia a unidades de superficie, 6,15), y se pide hallar el diámetro de las ciudades nueva y antigua.

Este sencillo ejercicio planteado a los aprendices de escribas (en el que se utilizará para nuestro $\pi$ actual el valor 3 ), aparentemente, respondería a necesidades prácticas propias de las sociedades agrícolas desarrolladas entre el Tigris y el Eúfrates, en concreto, a la planificación urbanística. La realidad, sin embargo, es muy diferente: jen Mesopotamia todas las ciudades tenían planta rectangular (prácticamente cuadradas en su mayor parte)! El problema, como tantos otros que pueden encontrarse en las numerosas tablillas que se conservan, no tenía ninguna aplicación en la vida real. Sin duda, se plantea por el interés intrínseco que tiene su contenido matemático; en consecuencia, la única utilidad que busca el maestro babilónico que lo inscribió sería de índole pedagógica: contribuiría a la formación matemática de los alumnos de su escuela.

Son los ejemplos de este tipo -muchos, por el elevado número de tablillas halladas-, junto con otros escritos en algunos papiros egipcios de la misma época -muy pocos, por el casi testimonial número de papiros conservados-, los que hacen que el entusiasmo que generan los sorprendentes hallazgos de las antiguas civilizaciones oculten lo que desde hoy es una realidad: solamente contienen cálculos numéricos concretos (o referidos a unas medidas particulares presentes en figuras, objetos, terrenos, etc., de formas geométricas determinadas) y todavía falta mucho tiempo hasta que los intereses conceptuales propios de los métodos subyacentes den lugar a enunciados teóricos generales (Bunt et al. 1988). En suma -y como veremos y justificaremos más adelante- perte- 
necen a unos momentos en la evolución de lo matemático que distan de ser científicos.

Pero las realizaciones matemáticas de cada una de las grandes civilizaciones de la Antigüedad no pueden verse -únicamente- en el seno del organismo cultural intrínseco sujeto a evolución, en analogía biológica tal como nos presenta Spengler en La decadencia de Occidente. El estudio de «lo matemático» de cada pueblo solamente puede hacerse en el marco de la evolución histórica general de este ámbito del saber humano. Cada «cultura» spengleriana ha matematizado hasta donde sus limitaciones intrínsecas le han permitido, pero la valoración de sus logros sólo puede hacerse en el contexto de la matematicidad potencial que todos los pueblos, en conjunto, han ido descubriendo y haciendo realidad.

Aunque discutible desde diferentes puntos de vista, el libro de Spengler (1923) ${ }^{2}$, repleto de ideas e hipótesis historiográficas, resulta sumamente valioso. En el presente artículo -mucho más modesto y, lógicamente, con muchas menos pretensiones- también aventuraremos algunas con ánimo de aportar una pequeña contribución al estudio de la problemática histórica y conceptual en su relación con la educación matemática.

\section{Una primeras perspectivas históricas sobre la naturaleza de la Matemática}

En el ejemplo babilónico precedente, en todo caso, se presentan dos aspectos de la Matemática que han tenido una importancia capital al organizar su transmisión desde entonces hasta nuestros días: «lo matemático» que se enseña por necesidad, contrapuesto a «lo matemático» como culminación del puro placer intelectual que sólo manifestará su proyección utilitaria como consecuencia. Estas perspectivas posibles conducen a la pregunta acerca de qué tipo de contenidos deben constituir la formación -general- de unos ciudadanos que distarán mucho de ser matemáticos profesionales y que van a ser -todo lo más-simples usuarios en su vida cotidiana de las herramientas que les proporciona esta Ciencia.

En paralelo e íntimamente relacionado con lo anterior, también existen dos puntos de vista sobre el origen histórico de la Matemática -en general, o, más en particular, de la Geometría-, los apuntados por Herodoto y Aristóteles, que no por haber sido citados tantas veces, hasta el punto de haberse convertido en tópicos, desaconsejan que los traigamos a estas páginas, pues ilustran sendas visiones acerca de la propia natu- 
raleza histórica de la disciplina. Veámoslo con cierto detalle (Fauvel y Gray 1997).

En su Historia (II, 109, escrita a mediados del siglo V a.C.) sitúa Herodoto su opinión acerca del origen de la Geometría al describir el sistema de reparto de las tierras, adoptado por el faraón, entre todos los egipcios sobre el que basar el cobro de los correspondientes impuestos. La necesidad de que las lindes originales de los terrenos asignados fueran recompuestas tras las crecidas anuales del Nilo exigía disponer de un cuerpo de funcionarios, conocidos como «los tensadores de la cuerda»-la «cuerda» era el útil para estimar longitudes-, que midieran los campos en proporción a los cuales se cobrarían los tributos. De acuerdo con esta perspectiva, los agrimensores -literalmente, medidores de campos de cultivo-y, por tanto, las necesidades prácticas, constituirían la fuente original donde los griegos habrían aprendido el arte de la Geometría.

Frente a Herodoto se sitúa el punto de vista que Aristóteles expone en su Metafísica (981 20 -25, mediados del siglo IV a.C.). Aquí, en su estudio acerca de las diferencias entre las artes prácticas y las ciencias, explica que las segundas, que no están dirigidas a resolver las necesidades de la vida cotidiana, no se descubrieron hasta que las primeras no estuvieron firmemente establecidas. Éste, sabio multidisciplinar -como aquél, historiador-, sitúa el origen de las artes matemáticas en Egipto, pero en este caso atribuye ese origen a la «casta sacerdotal», custodios celosos de los templos e interlocutores de las divinidades y, por tanto, los únicos que podían permitirse disfrutar del suficiente tiempo libre como para dedicarse a estas tareas ociosas que constituían los saberes geométricos ${ }^{3}$.

Por supuesto, entre los matemáticos de la Grecia clásica, pertenecientes al pequeño porcentaje de habitantes del mundo helénico con naturaleza de ciudadanos (el resto -la mayoría de la población-, extranjeros, esclavos, metecos, etc., no tenían esa consideración) es el último punto de vista el que prevalecerá. Ilustrativo de ello es el tratamiento de otro campo distinto del de la Geometría, el de lo numérico, en el que los griegos distinguen claramente dos ámbitos. En primer lugar, la Logísti$c a$, identificable actualmente con la Aritmética elemental, era propia de los mercaderes, de aquellos que tenían necesidad de utilizar los números y las operaciones realizables con ellos en la vida cotidiana. Lógicamente, para un pueblo de comerciantes como el griego resultaba imprescindible la instrucción del numeroso personal en las escuelas de contables. Frente a la «necesaria» Logística, ocupación de matemáticos verdaderos sería la «inútil» Aritmética, identificable hoy con la Teoría de números. Obvia- 
mente, los ciudadanos filósofos evitarían ocuparse de ninguna de las tareas -aquéllas-impropias de su clase, a la que sí corresponderían estas últimas, que serían las que se enseñasen en Academias y Liceos.

\section{La naturaleza científica de la Matemática}

A los efectos que interesan a estas páginas y, por tanto, prescindiendo de otras consideraciones -simplificando-, una Ciencia -en particular, la Matemática- es un conjunto de teorías científicas. Del mismo modo, una teoría científica es un sistema hipotético-deductivo, es decir, un conjunto de enunciados concatenados por las leyes de la Lógica -los teoremas-, que parten de unos primitivos que se admiten sin demostración -los axiomas-, y que se refieren a un conjunto de conceptos primarios indefinidos, cuya existencia se postula y admite, o a los definidos a partir de ellos (González Redondo 1993).

Cabe preguntarse, por tanto, en qué momento los desarrollos matemáticos adquirieron naturaleza científica y, por tanto, cuándo nació la Matemática. La cuestión no es baladí. Determinar qué es la Matemática resulta requisito imprescindible para organizar su enseñanza, pues dependiendo de qué concepto se tenga -o adopte- de la disciplina los enfoques docentes pueden diferir enormemente. En este sentido, el estudio histórico aporta algo de luz al tema.

De acuerdo con este enfoque, nuestra disciplina nacería en el momento en que se formulase la primera teoría matemática (es decir, la primera organización axiomático-deductiva de enunciados matemáticos), instante a partir del cual puede considerarse que ha alcanzado un estado científico ${ }^{4}$. En ese momento en el que surgiría un objeto historiable, en el que comenzaría su Historia, en consecuencia, la enseñanza de lo matemático experimenta -necesariamente- un cambio revolucionario. Veamos cuándo sucede esto.

De acuerdo con lo que conocemos hoy, y a pesar de diferentes menciones a otros autores anteriores, el primer ejemplo de utilización sistemática del método axiomático-deductivo lo constituyen los Elementos de Euclides de Alejandría. Con este tratado (este hito histórico) lo matemático adquiriría por primera vez carácter científico. Con él nacería la Matemática como Ciencia y comenzaría su Historia. Como escribe Luis Vega (1991): «No suele ocurrir que un solo tratado funde de una vez por todas una disciplina científica; aún es más extraño que además represente por más de veinte siglos el espejo y la norma del rigor de ésa y otras ciencias de la misma familia». 
Comienza Euclides con la relación de 23 «definiciones» (realmente caracterizaciones de conceptos primarios indefinibles, verdades inmediatas y evidentes para el alejandrino) de los objetos matemáticos que constituyen el punto de partida de la que se ha venido en considerar «axiomática material», sobre los que predicarán las diferentes proposiciones que compondrán los 13 libros del tratado: punto, línea (segmento rectilíneo), extremos de la línea, superficie, ángulo, etc. Continúa dividiendo los axiomas de su Geometría en dos grupos: «postulados» o axiomas propiamente geométricos, $\mathrm{y}$ «nociones comunes» $\mathrm{o}$ axiomas de validez universal que se podrían aplicar a todas las disciplinas a las que se quiera dar carácter científico. Hoy los denominaríamos, respectivamente, "sistema de axiomas no lógicos» $\mathrm{y}$ "sistema de axiomas lógicos» (Martínez 1986). El resto del primer libro corresponde a las proposiciones que, referidas a los objetos definidos, pueden demostrarse recurriendo únicamente a los axiomas admitidos. De ellas, 14 son realmente «problemas» (un objeto geométrico a construir) y 34 «teoremas» (asertos que se deben establecer acerca de alguna propiedad de los objetos definidos o construidos).

Los otros doce libros -desde la perspectiva actual, capítulos de un mismo tratado- comenzarán con las definiciones de nuevos conceptos (en conjunto habrá un total de 132 definiciones) a los que se referirán las nuevas proposiciones (465 en el total de los 13 libros): 2 problemas y 12 teoremas en el Libro II, 5 y 32 -respectivamente- en el Libro III, etc.

Obviamente los Elementos no surgen de la nada, pero desconocemos prácticamente tanto sus precedentes matemáticos como las circunstancias en las que se escribieron, por no insistir en la carencia completa de datos acerca del propio Euclides. Hoy se admite que constituyen la recopilación de numerosos enunciados propiamente matemáticos que no se habían reunido en un edificio sistemático hasta entonces, pero, sobre todo, se destaca (Heath 1956), y en esto se coincide con sus primeros comentaristas: a) lo certero en la selección de los problemas y teoremas que integra en el sistema, puesto que solamente incluye, de entre las enormes posibilidades a su alcance, aquellos resultados pertinentes para la construcción de elementos; y b) la variedad y riqueza de los métodos de demostración empleados.

Desde el punto de vista didáctico, como reconoce en sus comentarios Proclo, las perspectivas para la valoración de los Elementos crecen: a) en ellos se busca la claridad y la concisión, eliminando todo lo superfluo que dificulta la adquisición del conocimiento; b) se pretende que el estudiante que se aproxime al tratado obtenga una intelección precisa del conjunto de la materia; en suma, c) el autor no sólo pretende enseñar Geo- 
metría (y Aritmética), sino que intenta formar a los lectores en cómo construirla y aprenderla.

Pero que nadie busque (porque no lo encontrará) ninguna «finalidad» al margen de la propiamente matemática en Euclides. No se detecta adscripción alguna a posibles escuelas filosóficas, solamente desarrollos matemáticos. No existe aplicabilidad (potencial), mucho menos aplicación a ningún otro ámbito científico -si lo hubiera- o técnico, como la Astronomía, Óptica o Geografía, que podían haber sido, si no desarrollados, sí mencionados. Los Elementos son sólo, pura y llanamente, Matemática, recogida, eso sí, de las diferentes tradiciones.

\section{Categorías históricas, historiación y enseñanza de la Matemática}

La búsqueda de algunos «hitos singulares» facilita los cortes históricos, los instantes delimitadores de períodos o fases. En el plano usual de la historia tradicional los «hitos» de ordinario militares o guerreros son los determinantes, quizás por su concreción cronológica; en consecuencia, fácilmente utilizables como «límites» para la historiación. No sucede así, lógicamente, en las historias científicas y técnicas: la publicación de un libro científico tiene normalmente muy poco valor social y, por tanto, casi nada de trascendencia histórica desde su respectivo presente ${ }^{5}$.

La perspectiva histórica de que disfrutamos es suficientemente amplia para que pueda afirmarse que tratados como el de Euclides se convierten en fundadores, en punto de inicio de la Historia de la Matemática, que podrá o no hacerse coincidir, en la periodización de sus desarrollos posteriores, con las etapas que se han establecido en la Historia universal: Antigüedad, Edad Media, Modernidad, Edad Contemporánea. Conjuntamente con ello, la utilización de los prefijos de antelación "pre» (previo, pero de naturaleza distinta al lexema cuya carga semántica complementa) y «proto» (previo, pero de la misma naturaleza) facilitarán tanto la división en etapas -Prehistoria y Protohistoria- de la evolución del mundo de «lo matemático» hasta su constitución como Ciencia, cuanto la ubicación de los desarrollos de acuerdo con su momento histórico ${ }^{6}$.

En tanto que prefijos de antelación también pueden utilizarse no sólo en sentido temporal, sino también conceptual. Así, previa a la existencia de la Matemática como Ciencia, pero conformada por conceptos, métodos y desarrollos de naturaleza propiamente matemática, lo que existiría sería Protomatemática. El conjunto de descubrimientos que conducirán directamente a la construcción de los conceptos matemáticos, con algo 
propio de los sentidos que tendrán posteriormente, pero anteriores a toda consideración teórica general conformarían la Prematemática.

Análogamente, los conceptos matemáticos pueden presentarse, explicarse o enseñarse de diferentes modos. En primer lugar, científicamente, es decir, integrados estrictamente en el formato axiomático-deductivo de la teoría a la que pertenecen: sería una Presentación matemática. Pero también se pueden plantear los conceptos abstractos en un orden y con una interrelación entre ellos no sometidos al formato teórico: en este caso se trataría de una Presentación protomatemática. Finalmente, si el enfoque adoptado se limita a ejemplos o aplicaciones a métodos concretos, en la que conceptos y teorías quedan solamente subyacentes, estaríamos ante una Presentación prematemática.

Cabe preguntarse, por tanto, si es posible fijar otro «hito»-previo a los Elementos- que permita dividir en dos etapas claramente diferenciadas la evolución histórica de los conceptos matemáticos hasta su constitución como Ciencia, una en la que los desarrollos tengan un carácter claramente científico -la inmediatamente precedente de Euclides-, y otra, previa y necesaria para las formulaciones posteriores, pero que debe distinguirse por la naturaleza aún no científica de las realizaciones ${ }^{7}$.

Este «hito» puede ser la formulación, durante la primera mitad del siglo VI a.C., por parte de Tales de Mileto -pues así se le atribuye y reconoce-, de cinco enunciados acerca de propiedades generales de algunas figuras geométricas, supuestamente los primeros con estas características de la Historia (Boyer 1986):

1. Todo círculo queda dividido en dos partes iguales por un diámetro.

2. Los ángulos de la base de todo triángulo isósceles son iguales.

3. Los ángulos opuestos por el vértice que se forman al cortarse dos rectas son iguales.

4. Si dos triángulos son tales que dos ángulos y un lado de uno de ellos son, respectivamente, iguales a dos ángulos y un lado del otro, entonces los dos triángulos son congruentes.

5. Todo ángulo inscrito en una semicircunferencia es un ángulo recto.

Atribuirle también unas posibles demostraciones de estos teorema resulta exagerado, pero, en cualquier caso, por el carácter general puramente teórico de los enunciados, puede considerarse que Tales constituye, el «hito» buscado. Con él puede hacerse el corte histórico que buscábamos. Todo lo anterior a él pertenecería a la Prehistoria de la Matemática. Todo lo comprendido entre Tales y Euclides constituiría la Protohistoria de la Matemática.

Como parece natural, este enfoque historiográfico de «hitos» que producen cortes podría ampliarse a la periodización de la evolución históri- 
ca de todas y cada una de las ramas de la Matemática y a la del resto de los ámbitos científicos, con los ajustes necesarios y las consecuentes implicaciones para la docencia. Así, en Biología Darwin puede utilizarse como origen de la Historia de esta Ciencia, situando entre Leeuwenhoek, Hook, Linneo o Lamarck -justificando la elección- el comienzo de su Protohistoria. En Química Lavoisier y Mendeleiev también resultan especialmente significativos y podrían representar las oportunas rupturas. Para algunos fue Lyell quien intentó sentar las bases de una Geología realmente científica, pero ya Stennon, Werner y Hutton habían aportado nuevos aires a esta disciplina. Etc.

\section{Protomatemática. Protohistoria de la Matemática}

La idea de «demostración» que aparece por primera vez en Tales -si así lo admitimos- o, en cualquier caso, poco después en Pitágoras -si hacemos caso al resumen de Proclo- está en directa relación con la percepción de la posibilidad de conceptualización de lo verdadero, de la certeza, y a la posterior y generalizada aceptación incontestable de ella por todos los interlocutores. Esta posibilidad aporta un nuevo carácter a las atribuciones del conocimiento humano, al conceder a la Filosofía lo que hasta ese momento solamente se admitía en el mundo de la religión o el mito.

Así, llegado el siglo IV a.C., el de Platón y Aristóteles, probablemente gracias a la contribución matemática de los pitagóricos y a la filosófica de los pensadores del siglo anterior, fue posible hacer la distinción entre aquello de lo que podía haber conocimiento cierto, episteme, lo inmutable, y el mundo de lo opinable, doxa. Los ámbitos de ese conocimiento cierto, las Ciencias, solamente eran los cuatro mathemata, etimológicamente, «lo que se aprende», «lo que puede ser aprendido»: Aritmética, Geometría, Astronomía y Música.

La naturaleza de los silogismos, el formato axiomático-deductivo latente $\mathrm{o}$-probablemente- presente en los trabajos de los matemáticos del siglo IV a.C. (Teodoro, Teeteto, Eudoxo, Menecmo, ...), la estudiará monográficamente Aristóteles en sus tratados de Lógica (Órganon), sobre todo en sus Analíticos posteriores. Incluso las definiciones euclídeas debían estar ya planteadas de forma casi idéntica a como aparecen en los Elementos, como puede observarse en la Metafísica del filósofo macedonio. Y es que entre los geómetras anteriores a Euclides ya debía haberse alcanzado un acuerdo suficiente sobre las características de la demostración matemática, que en sus líneas generales son las que se recogen en 
los únicos trabajos completos que se conservan anteriores al siglo III a.C., Sobre la esfera en movimiento y Sobre ortos y ocasos de Autólico de Pitania (Heath 1981).

Los Elementos suponen la culminación de una tradición griega de elaboración de tratados elementales de los que no se conserva ninguno. Si seguimos (Rey Pastor y Babini 1985) el resumen -recogido por Proclo- de la Historia de la Matemática, que se supone escribió un discípulo de Aristóteles llamado Eudemo de Rodas (fl.c. 320 a.C.), el primer autor que escribió un libro de elementos fue Hipócrates de Quíos (470-400 a.C.). Algunos años después León -matemático más joven que Platón y mayor que Eudoxo de Cnido- presentó otro tratado prestando más atención al número de elementos y a su utilización en las pruebas. Finalmente, un tercer autor, probablemente del entorno de la Academia de Platón, Teudio de Magnesia, escribió la última recopilación de elementos anterior a Euclides -y poco más sabemos de él- que mejoraba el orden de las deducciones y aportaba una forma más general a resultados que hasta ese momento sólo habrían tenido un alcance restringido. En todo caso, de acuerdo con Proclo, Hermótimo de Colofón -algo más joven que Euclideshabría sido descubridor de muchos de los elementos ${ }^{8}$.

Pero conviene destacar que en el mundo helénico la expresión «elementos» tenía diferentes significados:

a) Recopilaciones de algunos conocimientos primordiales determinados.

b) Aquellas proposiciones que desempeñaban un papel esencial en la posibilidad de organización deductiva de otros desarrollos.

c) Todo aquello (problema, lema o teorema) que puede ser utilizado para establecer algún nuevo resultado. Pero en su sentido más propio «Elementos» designa aquel grupo de asunciones y proposiciones que tienen el carácter de principios en la ligazón deductiva de un determinado campo de conocimientos.

Algunos años después de publicado el magno tratado euclídeo, el mayor matemático de la Antigüedad, Arquímedes de Siracusa, precisaba en El Método lo que desde el siglo anterior venía destacándose: el conocimiento matemático se acrecienta mediante el complemento mutuo de los procesos de análisis y de síntesis, de inducción y de deducción; las presentaciones sintéticas que aportan la solidez lógica al objeto de estudio no son el resultado final de ensayos, rectificaciones, experiencias, analogías, abstracciones aisladas, etc.

Para la formación matemática, para generar el conocimiento matemático en los escolares, parece natural seguir el mismo proceso: a) germinar los conceptos abstractos a partir de numerosas observaciones, experiencias 
e intuiciones (Puig Adam 1960), pues las síntesis a priori bloquean las aptitudes analizadoras de los estudiantes; b) transmitir la naturaleza científica (axiomático-deductiva) de la disciplina a partir de generalizaciones concretas y teoremas particulares, que se van engarzando en el entramado lógico de las teorías, de modo que los alumnos alcancen las síntesis que plantearán los profesores; y c) integrar las motivaciones prácticas iniciales y las aplicaciones posteriores a la realidad concreta con el carácter lúdico del disfrute intelectual. En suma, recuperando los términos introducidos antes, llegar a la Matemática desde la Protomatemática.

\section{Prematemática. Prehistoria de la Matemática}

La visión que la sociedad tiene de la Matemática es la de que se trata del campo del saber que se dedica al dominio de lo numérico y del cálculo, y que, por tanto, los matemáticos son poco más que los guardianes del saber contable. Esta perspectiva tan mutilada es -ciertamente- muy próxima a lo que comenzaba para los griegos clásicos siendo la Logística. Pero aún más, hoy, entre técnicos e ingenieros de las diferentes ramas y entre científicos aplicados de las diferentes disciplinas, la Matemática es poco más que un lenguaje simbólico útil para expresar las fórmulas que precisan y necesario para los numerosos cálculos que deben realizar (Muñoz 1995).

Sin embargo, lo más grave es que este sentido tan parcial de la Matemática es el que desde diferentes pedestales pedagógicos se ha pretendido generalizar, el de simple conjunto de herramientas que debe conocer todo ciudadano y en las que hay que instruir en la Escuela. Con este planteamiento, las características abstractas de la materia, cuya utilidad y/o aplicabilidad resultan inverosímiles para los estudiantes que la reciben, ocasionan el bien conocido bloqueo y rechazo y logran el temido y denostado fracaso escolar.

En las tablillas de los mesopotámicos o los papiros de los egipcios no se encuentra nada (por lo menos, de acuerdo con los datos que proporciona la Arqueología matemática en su estado actual) que pueda parecerse a una demostración de una proposición general. Todo lo más se «comprueba» la validez de un resultado concreto obtenido para un problema particular planteado, en una serie más o menos amplia de problemas de un mismo tipo. Ni unos ni otros, los más cultos de entre los antiguos, ni siquiera tuvieron la necesidad o disfrutaron la oportunidad de enunciar ningún método general. Parecía bastarles la validez sistemática de los resultados individuales en su contraste con la experiencia. 
Eso no impide que nos sorprenda el nivel matemático de algunos problemas de los papiros Rhind y de Moscú o de escritos como Plimton 322, tablilla mesopotámica del período babilónico antiguo (entre 1900 y 1600 a.C.) de la colección de la Universidad de Columbia. En esta última (Fauvel y Grey 1987), aunque conservada sólo parcialmente, se encuentran tabulados valores referidos a triángulos rectángulos que un análisis exhaustivo permite concluir que corresponden a la $\sec ^{2} \alpha$, realmente valores de $c^{2} / b^{2}$, si $b$ es el cateto contiguo al ángulo $\alpha$ y $c$ la hipotenusa del triángulo ${ }^{9}$.

Una parte muy importante de la Matemática elemental, de los contenidos, técnicas y métodos habituales matemáticos de la Educación Primaria, tiene más semejanzas con lo que encontraremos en las civilizaciones más antiguas que con lo generalizado en las exposiciones de la materia desde la Grecia helenística. La relación de los ejercicios y problemas sacados de situaciones reales del medio circundante y con aplicabilidad en la vida práctica, su relación con otros ámbitos en los que tendemos a parcelar el conocimiento, puede acompañarse con la motivación hacia la comprobación, con la guía hacia el rigor de los procesos de razonamiento.

Si junto con el enfoque predominantemente prehistórico (o prematemático, en el sentido que estamos defendiendo en este trabajo), planteásemos un enfoque respetuosamente histórico en las asignaturas escolares, de modo que predominase la estima por lo científico de las ciencias, y del que trascendiera, además, la belleza de las aplicaciones que tiene en los diferentes ámbitos de la vida cotidiana, el rechazo difícilmente podría justificarse y el fracaso escolar probablemente empezaría a dejar de tener sentido. Como escribía Puig Adam (1960): «Si las principales teorías han tomado origen de problemas prácticos, no es menos cierto que los conceptos abstractos en ellas elaborados se enseñorean de nuestra mente, y al tomar carta de naturaleza en ella, proliferan, dando lugar a las más bellas construcciones intelectuales, cuyas aplicaciones futuras todavía son un misterio».

Por supuesto no es esa la tendencia generalizada. En la pedagogía matemática actual (y no sólo en ella) predomina la opinión de que las abstracciones son intrínsecamente frustrantes y que en la experimentalidad esencial y omnipresente -que consideran compatible con la naturaleza de esta Ciencia-radica el fundamento de lo que debe ser la instrucción matemática edificante y motivadora y la que contribuirá a hacer cultos a los escolares. Se proporciona sólo la instrucción de técnicos, cuasi-ingenieros o pseudocientíficos aplicados sin Ciencia. En el mundo de la especialización impuesta durante el siglo XX no se conciben las mentali- 
Sobre la naturaleza histórica de la Matemática

dades universales, los individuos con una formación integral general. No se entiende que la Historia nos proporciona una clara idea de qué es ser culto; y que aquel que realmente lo es tiene la capacidad para aplicar su cultura, las Ciencias aprendidas, en contextos prácticos.

Seguía escribiendo Puig Adam (1960): «El doloroso dilema es éste: O la especialización intensa, sinónimo de esclavitud angustiosamente aniquiladora, o el enciclopedismo libre, multifacético, de extensa vibración, pero, por lo mismo, carente de profundidad». Esto, referido al ámbito universitario, exigiría considerar la enorme proliferación numérica de profesores e investigadores que permite eludir el modelo único. Pero situado en los niveles educativo primario y secundario, comunes para la mayoría de la población, adquieren una perspectiva todavía más clara.

\section{Matemática, Modernidad y Neoclasicismo}

La Modernidad, esa Era que comienza en torno a $1600^{10}$ en la que se alcanzará definitivamente la superación de los logros de la Antigüedad tras los siglos de la Edad Media ${ }^{11}$, tendrá entre sus ingredientes constitutivos las posibilidades que aporta la nueva Matemática del siglo XVII. El punto de partida debe situarse un poco antes del comienzo de la centuria, en la Introducción al Arte Analítica (1591) de Vieta (González Redondo 1995), pero se cimenta en la Geometría (1637) de Descartes, centrado en el desarrollo de la notación simbólica para las relaciones magnitudinales en las igualdades entre medidas que suponen las ecuaciones (González Redondo y Redondo Alvarado 1996). Y las que suelen reconocerse como principales novedades -las capitales- tienen mucho que ver con este desarrollo: Análisis algebraico, Geometría analítica y Cálculo infinitesimal.

Pero si para el acervo cultural universal hay una aportación capital de la nueva época que en el siglo XVII comienza, ésta es la posibilidad de matematización, por primera $\mathrm{vez}^{12}$, en formato axiomático-deductivo (escritos, como se decía, more geométrico), de algunos aspectos de la realidad; es decir, el nacimiento de las teorías físicas, apuntado por Galileo para la Cinemática en sus Discursos sobre las dos nuevas Ciencias (1638) -todavía prácticamente en el ámbito sólo de la Matemática- y culminado en los Principios matemáticos de la Filosofía Natural de Newton (1687), donde la introducción de los conceptos de masa y fuerza dan entrada a la Dinámica en el dominio de la Matemática. El siglo siguiente, el de Euler, D’Alembert, Lagrange y Laplace, supondrá el de la culminación del edificio newtoniano en un proceso en el que la redacción retórica 
se sustituye por la expresión algebraica y los desarrollos geométricos por los analíticos (González Redondo 2000).

Desde esta nueva perspectiva se podían construir modelos matemáticos del mundo de lo sensible que, en su contraste con la Naturaleza, parecían «verdaderos» ${ }^{13}$. La Matemática se constituía -no sólo, pero sí de manera importante- en una herramienta para el conocimiento «cierto» -sucesivamente- del movimiento de los cuerpos, de los fenómenos termológicos, eléctricos, ópticos o magnéticos. A todo ello contribuiría en no poca medida durante el siglo XVIII un Análisis matemático al que no se le exige rigor teórico, sino utilidad a la hora de proporcionar las herramientas para aprehender científicamente la Realidad.

Como escribiría Gauss en el siglo siguiente, ilustrando su época (Muñoz 1995): «Siempre hay personas que no saben nada de la nobleza de las verdades eternas y de su divina belleza y, por ello, sólo dan valor a las investigaciones matemáticas por su utilidad en el dominio de las ciencias aplicadas; los desarrollos anteriores [desarrollos en serie aplicables en Mecánica celeste] tendrán la utilidad de hacer agradables a tales gentes nuestras investigaciones».

$\mathrm{Y}$ como si todo esto hubiese ocurrido en fechas tan próximas a nosotros que -por la falta de perspectiva histórica- nos hubieran pasado desapercibidas la motivación, gestación, formulación y desarrollo (no sólo) del Análisis (pero sí especialmente), de estas circunstancias se harían eco hace unos años los redactores del currículo de la Educación Secundaria (ya pre-universitaria) al hacer que en todas y cada una de las opciones o itinerarios la Matemática se convirtiera únicamente en un lenguaje auxiliar para aquellos que van a estudiar una disciplina científica (Física, Química, Biología o Geología), una ingeniería (de Caminos, Industrial, Telecomunicaciones, etc.), alguna ciencia social (Económicas, Empresariales, etc.), una carrera de la rama sanitaria, etc., y no se generalizase como un saber valioso per se.

Un momento singular, «hito» histórico en la evolución de la Física y la Matemática, punto de origen del Neoclasicismo matemático ${ }^{14}$, lo constituye la obra de Fourier, quien no sólo aporta con su Teoría analítica del calor (construida entre la primera publicación de 1807 y la definitiva de 1822) la primera teoría de la Física-matemática en sentido actual, sino quizá, también, la primera aproximación al concepto contemporáneo del punto de partida de toda axiomatización, de todo sistema hipotético deductivo: el objeto de estudio primordial e indefinido, el calor, no se sabe qué es a priori ni para Fourier resulta necesario ni importa que se sepa (desde luego no será el tradicional «calórico» ni se atisba aún como «forma de energía»). En todo caso, su comportamiento se somete a la ley de 
la conducción que éste enuncia, prácticamente «definitoria», al mismo tiempo, de su naturaleza (González de Posada et al 1991).

La composición de los Elementos había constituido una obra de capital importancia tanto teórica como didáctica. En cuanto a lo primero, la recopilación de resultados demostrados estableció el modelo metodológico a seguir para sistematizar científicamente un cuerpo de conocimiento ${ }^{15}$. Por lo segundo, se había convertido en el libro de texto para los estudiantes de Geometría y Aritmética, no sólo ya del mundo helenístico, sino que por el carácter de «manual» de la obra -y adaptado no siempre convenientemente-, mantendría una posición central en la enseñanza secundaria de muchos países -entre ellos España- hasta finales del siglo XIX.

A medida que avanzaba el siglo XX el Cálculo Infinitesimal comenzó a generalizar su presencia en los Bachilleratos de los países desarrollados. Ciertamente resultaba imprescindible para las formulaciones de una materia como la Física, pero se explicaba (y se sigue explicando) al margen de ella, prescindiendo orgullosamente de sus orígenes y sentidos creacionales.

Pero en los momentos iniciales del siglo XIX la fundamentación de todas y cada una de las ramas de la Matemática se asumía como objetivo pendiente. Estaba claro que incluso la Geometría euclídea necesitaba una profunda revisión. La época que hemos conjeturado que comienza con Fourier puede caracterizarse como la de consecución de esa tarea: los matemáticos aportaron los conceptos abstractos y las organizaciones teóricas como ejercicios intelectuales al margen de la que ya era remota idealización simbólica de la Realidad sensible. Se formaliza el Análisis (el concepto de número real, el de función real de variable(s) real(es), el de continuidad, límite, derivada, ...), el Álgebra se establece como estudio de las estructuras, se ordenan las nuevas geometrías ubicando -muy depurada- la que presentó Euclides en un marco general con las demás... hasta establecer sólidamente la Aritmética del número natural ante el cambio de siglo (Boyer 1986).

En suma, la Matemática en su conjunto (y sus partes) alcanzaba rango científico (tras sus estadios protocientíficos) bajo el formato de los sistemas axiomáticos formales, en los que los conceptos primarios perdían toda conexión con las verdades abstraídas de la Naturaleza. Eso sí, para encontrar posteriores aplicaciones cuando los estudiosos de la Realidad tenían que echar mano del repertorio de Matemática -ahora sí, intrínsecamente inútil en modo apreciable- puesto a su disposición para ser utilizado oportunamente. Toda la Matemática podía comenzar a ser objeto de la Historia, sus partes pasaban a ser objetos historiables. 


\section{En torno a la Matemática contemporánea. Consideraciones históricas y educativas finales}

Desde el punto de vista del estudio filosófico del conjunto de las Ciencias, el período que hemos denominado Neoclasicismo terminaría con el siglo XIX, identificándose este cambio con la ruptura con el mundo newtoniano-kantiano que supone la revolución relativista. Para no cambiar demasiadas denominaciones todavía generalizadas, el instante actual que nos toca vivir debe considerarse nuestra Edad Contemporánea, y su comienzo puede fijarse en un momento, en torno a 1930, y una serie de «hitos» delimitadores del corte que describiremos a continuación.

La Metaciencia que gobernó la formulación científica de la Geometría por Euclides, la Mecánica por Newton y los diferentes campos, tanto de la Matemática como de la Física hasta comenzar el siglo XX, se caracterizaba por la estricta independencia y nítida separabilidad de los conceptos primarios indefinibles, puntos de partida de las teorías de una y de otra. Sin embargo, el análisis de la formulación de la Relatividad General (cerrada con la eliminación de la constante cosmológica por Einstein tras la hipótesis de Hubble de 1929 sobre la expansión del Universo) impone una nueva visión metacientífica: los conceptos magnitudinales primordiales no sólo son no separables, sino que están en íntima e intrínseca «respectividad» entre ellos (González de Posada 2001). Sin embargo, la Matemática que usa sigue respondiendo a algunas presuposiciones filosóficas que ya se asumían en la época de Aristóteles. Dado que la Geometría, el Álgebra y el Análisis que se siguen utilizando responden a unas presuposiciones clásicas, caben serias dudas acerca de que la Matemática de la respectividad relativista responda a la estructura lógica de los formatos axiomático-deductivos.

Por ejemplo, la masa de los cuerpos (magnitud primaria y, por tanto, indefinible en la Mecánica Clásica) no es ya que dependa de la velocidad a la que se mueve el cuerpo (magnitud secundaria, definida por la derivada del vector desplazamiento respecto del tiempo), es que puede «definirse» (¿dejando de tener el carácter de concepto «primario» de la teoría?) en función de la masa del cuerpo en reposo $m_{0}$, la velocidad del cuerpo $v$ y la velocidad de la luz c (González de Posada y González Redondo 1994).

Por otro lado, la aparición de modelos no sólo no deterministas, sino intrínsecamente probabilistas para la aprehensión matemática de la Realidad, en Economía, Medicina, Sociología, etc. y, sobre todo, con las Mecánicas estadísticas comenzadas a alumbrar en el último tercio del siglo XIX, llevará durante las primeras décadas del XX a una «incerti- 
Sobre la naturaleza histórica de la Matemática

dumbre» matemática desconocida en los modelos causales clásicos habituales hasta entonces.

Además, el convencimiento de que la materia es discreta hace que la matemática del número real no sirva (hay que prescindir de la continuidad de la recta real, de los conceptos de función real continua, derivable, etc.). $\mathrm{Y}$ a esto hay que añadir, entre otras cosas, que la discretización tampoco es la de los números enteros. Ciertamente, aunque la Física cuántica, la que pretende dar cuenta de estos temas, es propiamente matemática (y no es fácil que alguien crea que la Naturaleza pueda parecerse a las formulaciones que desde aquélla se presentan) existe el convencimiento, aunque en torno a 1930 se consideraba firmemente establecida, de que todavía le falta desarrollar toda su matematicidad.

Pero lo que trastocará más profundamente el panorama de la formulación matemática serán los sobrecogedores hallazgos de Gödel, recogidos en «Sobre las proposiciones formalmente indecidibles de los Principia matemática y sistemas relacionados», de 1931, en el que presentará su Teorema de incompletitud. Éste afirma que si una teoría formal T que abarca la teoría de los números enteros es consistente, entonces es incompleta, es decir, existe un enunciado «S» tal que ni «S» ni «no $\mathrm{S}$ » pueden ser demostrados en $\mathrm{T}$; como uno de los dos enunciados «S» o «no $\mathrm{S}$ " tiene que ser verdadero, existe una proposición verdadera de la teoría que no es demostrable y, por lo tanto, es indecidible. En consecuencia, no puede ser demostrada la consistencia de un sistema lo suficientemente amplio como para abarcar la aritmética de los números enteros.

El único ingrediente que hacía intrínsecamente diferente a la Matemática del resto del conocimiento científico, la validez incontestable de sus resultados, se diluía. Había comenzado lo que Kline (1985) denominaría «desastres». Hasta en el propio método axiomático-deductivo, esa aproximación "perfecta» a la exactitud del conocimiento, se encontraban fallos.

Para finalizar, podemos afirmar que, indudablemente, debemos estar en crisis. Han transcurrido 70 años desde aquellos entonces y aún no sólo no constituyen objetos de la Historia sino que todo es presente. Y el mundo educativo lo recibe directamente. Sin que existan firmemente establecidos y generalizados unos objetos, metodologías, estructuras internas y consistencia comunes, es decir, unas caracterizaciones de disciplinas como las Matemáticas ${ }^{16}$ (que pierden la singularidad deseable en beneficio de una pluralidad aún no evitable) o como las Físicas (unas incompatibles con las otras), su transmisión y divulgación se ven dificultadas.

Pero la enseñanza de nuestra Ciencia está en manos del profesorado formado en la Universidad, al que se ha dado -en general- la más ma- 
temática de las presentaciones posibles: sucesión de abstracciones entrelazadas deductivamente, para las que se evita consciente y orgullosamente toda motivación respetuosa con la evolución histórica y a la que se le hurtan las perspectivas de las aplicaciones que de ella harán científicos de otros campos e ingenieros. En ellos recaerán, sin embargo, unas enseñanzas Primaria y Secundaria en las que las respectivas presentaciones serán esencialmente prematemáticas y protomatemáticas. Es legítimo, por tanto, plantearse cuestiones como desde qué punto de vista y con qué herramientas lo harán, a quién debe atribuírsele la responsabilidad por la situación en la que nos encontramos o a quién corresponde presentar soluciones.

Para complicar el panorama educativo, también podíamos referirnos a la íntimamente ligada desaparición de valores morales generales y claros en la Sociedad de este período intersecular que nos toca vivir. Pero la Realidad es azorante y esos son ya otros temas.

\section{Notas}

1 Trabajos clásicos con transcripciones de numerosos escritos matemáticos mesopotámicos, algunos de los cuales se seleccionan en el libro fácilmente accesible de Fauvel y Gray (1987), son los de Thureau-Dangin (1938) -en francés- y Neugebauer y Sachs (1945) -en inglés-.

2 Utilizamos la edición prologada por Ortega y Gasset en dos volúmenes, convertida ya en clásica. Puede compararse nuestra lectura de Spengler con la visión de Muñoz (1995).

3 Otros autores helénicos que se han referido a estas mismas cuestiones son Platón en Fedro (274 cd, comienzos del siglo IV a.C.) y Proclo en Sobre Euclides (I, siglo V d.C.)

${ }^{4}$ Lo que no quiere decir que la mayor parte de los restantes campos de la Matemática no tardaran muchos siglos en alcanzar ese estadio teórico.

5 Este enfoque historiográfico lo presentamos por primera vez en González Redondo (1995) y lo desarrollamos en González Redondo (2000), todo ello en el marco de la escuela de Fundamentos y Filosofía de la Ciencia del Prof. F. González de Posada. Algunos términos (y los conceptos que denotan) los tomamos de Américo Castro (1956) y los aplicamos, por ejemplo, a la Historia de la Matemática Española, en González Redondo y de León (2000), y a la Historia de la Matemática en general en González Redondo (2002).

6 Como parece claro, estas consideraciones, que aplicadas a la Historia de la Ciencia consideramos originales, constituyen una adaptación tomada de la Historia Universal, que reformulamos como sigue: la Historia del hombre comienza en el momento que se desarrolla la escritura; la Protohistoria discurriría desde que existe propiamente el hombre -la especie homo sapiens - hasta que se descubre la escritura; y Prehistoria sería todo lo anterior a la aparición de la especie. Periodización heterodoxa, sin duda, resulta sorprendente que la aparición del homo sapiens no se constituya en "hito" singular que proporcione el oportuno corte histórico, y se siga hablando de un período como el Paleolítico Superior que integra conjuntamente al hombre y a los homínidos.

7 Igual que no todos los pueblos de la Tierra alcanzaron la etapa histórica al mismo tiempo que mesopotámicos y egipcios, en el mundo griego entre el siglo III a.C. y el V d.C. 


\section{Sobre la naturaleza histórica de la Matemática}

será en el primer y único lugar en el que se alcance la etapa propiamente matemática, hasta que en algunos países de la Europa occidental pueda considerarse recuperado el nivel en torno al siglo XVII.

8 Llegados a este punto del artículo, quizá no esté de más precisar que queremos evitar la ilusión de reconstrucción finalista, y que pensamos que debe descartarse una supuesta trayectoria lineal de sistematización progresiva de los conocimientos matemáticos hasta la versión definitiva de Euclides, aunque éste se apoyase o partiese de otras aproximaciones precedentes.

9 Con un sentido próximo al nuestro, Boyer (1986) califica de "prototrigonometría" al contenido matemático de esta tablilla.

10 Tomamos la expresión del punto de vista de Ortega y Gasset. Ese año, entre otros acontecimientos, muere en la hoguera Giordano Bruno, se publica De Magnete de Gilbert y Kepler comienza a trabajar con Ticho Brahe. Ver González de Posada, F. (ed.): Cosmología. En torno a Galileo, 1993. Madrid: Amigos de la Cultura Científica.

${ }^{11}$ La calificará Spengler (1923) de "progresiva emancipación de los influjos antiguos, una liberación que ni siquiera fue deseada, sino obligada por hondas tendencias inconscientes».

12 Suponiendo que la Geometría griega clásica no deba ser considerada como la primera matematización o aprehensión matemática de la Realidad y, por tanto, la primera teoría física.

13 Con las «leyes» de (Galileo y) Newton se podrían predecir comportamientos físicos; por ejemplo, la existencia de planetas desconocidos que se irán «descubriendo» matemáticamente, no observacionalmente. Más adelante serán esas mismas leyes las que permitirán salir de la atmósfera terrestre, llegar a la Luna y, lo que es incluso más importante, volver después a la Tierra.

14 Utilizamos a nuestra libre interpretación las denominaciones establecidas hace ya demasiados decenios para los distintos períodos históricos, aunque no se nos escapa que podrían discutirse. Normalmente se denomina Postmodernidad a la era científica, filosófica y social que sigue al derrumbamiento de los presupuestos de la Modernidad con la formulación de la Relatividad General de Einstein y el desarrollo de las teorías cuánticas, época que trataremos en el parágrafo 7. Por otro lado, resulta necesario modificar otras manifestaciones de la historiografía usual, pues situar la contemporaneidad en el siglo XIX no parece aceptable. Optamos por considerar -y no parece descabellado- que el período que comienza con Fourier es la época Neoclásica, teniendo en cuenta los propósitos fundamentadores de los matemáticos que la caracterizan.

15 Sobre los intentos -infructuosos- de extender en su época la sistemática euclídea a otros dominios no propiamente matemáticos, como la Medicina (Galeno), puede verse Vegas (1991).

16 Uno de las contribuciones más recientes sobre la situación de la Matemática y su enseñanza en la actualidad es el «Borrador: Sobre la situación de la enseñanza de las Matemáticas", de la Comisión de Educación de la Real Sociedad Matemática Española. En Gaceta de la RSME, vol.4, $\mathrm{n}^{\circ} 3$ (2001).

\section{Bibliografía}

BOYER, C. B. (1986): Historia de la matemática. Madrid: Alianza. 
Bunge, M. (1967): Scientific Research. (2 vols.). New York: Springer-Verlag.

Bunt, N. H., Jones, P. S. y BEDIEnT, J. D. (1988): The Historical Roots of Elementary Mathematics. New York: Dover.

CASTRO, A. (1956): «Descripción, narración e historiografía». En Dos Ensayos. México: Porrúa.

Eves, H. (1997): Foundations and Fundamental Concepts of Mathematics. New York: Dover.

FAUVEL, J. y Gray, J. (1987): The History of Mathematics: A Reader. Milton Keynes: The Open University.

GILlINGS, R. J. (1972): Mathematics in the Time of the Pharaos. Cambridge (Mass.): M.I.T. Press.

GONZÁlez DE POSADA, F. (2001): La Física del siglo XX en la Metafísica de Zubiri. Madrid: Instituto de España.

GonZÁlez De PosAdA, F. y GonZÁlez Redondo, F. A. (1994): “Acerca de la no aplicabilidad lógica del Análisis Dimensional clásico a la Teoría de la relatividad". En Anuario Científico 1993 del Grupo de Análisis Dimensional, pp. 19-22. Universidad Politécnica de Madrid.

GonzÁlez de PosadA, F. y GonZÁlez Redondo, F. A. (1996): Blas Cabrera: Principios fundamentales de Análisis vectorial en el espacio de tres dimensiones y en el Universo de Minkowski (1912-1913). Madrid: Amigos de la Cultura Científica.

GonZÁlez de PosadA, F., GonZÁlez Redondo, F. A. y Redondo Alvarado, Ma D. (1991): "Las 'Remarques Gènèrales' en la Théorie analytique de la chaleur de Fourier». En Anuario Científico 1990 del Grupo de Análisis Dimensional, pp. 157-171. Universidad Politécnica de Madrid.

GonZÁlez Redondo, F. A. (1993): El Análisis Dimensional en la obra de Mario Bunge. Tesis Doctoral en Filosofía. Universidad Complutense de Madrid.

GoNZÁlez REDONDO, F. A. (1995): «Historia del Postulado General de Homogeneidad. En torno a F. Viete (1591)». En Anuario Científico 1994 del Grupo de Análisis Dimensional, pp. 167-174. Universidad Politécnica de Madrid.

GonzÁlez Redondo, F. A. (2000): Historia del Análisis Dimensional. Tesis Doctoral en Matemáticas. Universidad Politécnica de Madrid.

GONZÁLEZ REDONDO, F. A. (2002): «Un modelo historiográfico para las Ciencias. La evolución de la Matemática hasta su establecimiento como disciplina científica». Boletín de la Sociedad Puig Adam de Profesores de Matemáticas $\mathrm{n}^{\circ} 61$.

GonZÁlez Redondo, F. A. y Redondo Alvarado, Ma. D. (1996): «Las concepciones dimensionales en la obra de R. Descartes». En Anuario Científico 1995 del Grupo de Análisis Dimensional, pp. 109-115. Universidad Politécnica de Madrid.

GonZÁleZ ReDONDO, F. A. y DE LEÓN, M. (2000): “Aproximación a la Historia de las Matemáticas en España». En Gaceta de la Real Sociedad Matemática Española vol. 3, $\mathrm{n}^{\circ} 2$, pp. 363-370.

HEATH, T. (1956): The Thirteen Books of Euclid's Elements. (3 vols.). New York: Dover.

HEATH, T. (1981): A History of Greek Mathematics. (2 vols.). New York: Dover.

KLINE, M. (1985): Matemáticas. La pérdida de la certidumbre. Madrid: Siglo XXI.

MARTINEZ, M. (1986): "Los Orígenes del método axiomático-deductivo». En Historia de la Matemática hasta el siglo XVII, pp. 37-60. Madrid: Real Academia de Ciencias Exactas, Físicas y Naturales.

MuÑoz DfAZ, J. (1995): «Reflexiones acerca de la naturaleza e historia de las Matemáticas». Discurso de apertura del curso académico 1995-1996. Universidad de Salamanca. 


\section{Sobre la naturaleza histórica de la Matemática}

Neugebauer, O. (1969): The Exact Sciences in Antiquity. New York: Dover.

Neugebauer, O. y SACHS, A. (1945): Mathematical Cuneiform Texts. New Haven: Yale University Press.

Puig AdAM, P. (1960): La Matemática y su enseñanza actual. Madrid: Ministerio de Educación.

REY PASTOR, J. y BABINI, J. (1985): Historia de la matemática. (2 vols.). Barcelona: Gedisa.

SPENGLeR, O. (1923): La decadencia de Occidente. (2 vols.). Madrid: Espasa-Calpe.

Thureau-Dangin, F. (1938): Textes Mathematiques Babyloniens. Leiden: Brill.

VEGA REÑóN, L. (1991): «Introducción General» a Euclides: Elementos, pp. 7-184. Madrid: Gre los. 